\title{
Comparison of Three Nucleic Acid Amplification Tests for Detection of Chlamydia trachomatis in Urine Specimens
}

\author{
Charlotte A. Gaydos, ${ }^{1 *}$ Mellisa Theodore, ${ }^{1}$ Nicholas Dalesio, ${ }^{1}$ Billie Jo Wood,${ }^{1}$ and \\ Thomas C. Quinn ${ }^{1,2}$ \\ Johns Hopkins University, Baltimore, ${ }^{1}$ and National Institute of Allergy and Infectious Diseases, \\ National Institutes of Health, Bethesda, ${ }^{2}$ Maryland
}

Received 14 January 2004/Returned for modification 17 March 2004/Accepted 13 April 2004

\begin{abstract}
Traditionally, culture and immunoassays have been performed for the detection of sexually transmitted diseases, including Chlamydia trachomatis. However, these assays may often require invasive specimen collection methods, such as female cervical and male urethral swabs. Recently, nucleic acid amplification tests (NAATs) have been approved for testing for the presence of $C$. trachomatis in urine samples. Our objective was to compare the sensitivities and specificities of $C$. trachomatis detection in urine samples with three NAATs: the Abbott LCx (LCx), BD ProbeTec ET (ProbeTec), and Gen-Probe APTIMA Combo 2 (AC2). Urine specimens $(n=506)$ were collected from both symptomatic and asymptomatic males and females from various high school health clinics. Specimens were tested for $C$. trachomatis with the three NAATs, and a true-positive result was defined as any two positive NAATs. The $C$. trachomatis prevalence was $14.8 \%$ ( 75 of 506 samples). Of the 75 urine samples defined as true positives, LCx detected 72, ProbeTec 72, and AC2 detected 75. The sensitivities of $\mathrm{LCx}$, ProbeTec, and AC2 for C. trachomatis detection were 96.0, 96.0, and 100\%, and the specificities were 99.1, 100, and 98.8\%, respectively. Four of five samples that were positive with AC2 and negative with LCx and ProbeTec were found to be positive with an alternative target TMA-based NAAT, APTIMA $C$. trachomatis, suggesting that they may have been true positives. Two of four uniquely positive LCx samples available for subsequent testing were both found to be positive by Roche PCR. We found that the LCx, ProbeTec, and AC2 NAATs are highly sensitive and specific methods for the detection of $C$. trachomatis in urine specimens and can be recommended for noninvasive screening of $C$. trachomatis in urine.
\end{abstract}

Chlamydia trachomatis is the most prevalent bacterial sexually transmitted disease in developed countries (8). Because most infected individuals are asymptomatic (up to $50 \%$ of men and $80 \%$ of women), the actual number of reported cases represents only a fraction of the infected population (8). If we take this into account, it can be estimated that perhaps 3 to 4 million cases occur annually in the United States and that 89 million cases occur worldwide (8). The prevalence of $C$. trachomatis in woman under 25 years of age is very high (up to $30 \%$ ); hence, young sexually active women are the most at risk (3-5). If untreated, the infection may evolve into pelvic inflammatory disease and may result in serious sequelae, such as ectopic pregnancy and infertility $(6,8)$. Because the infection is easily treatable with antibiotics, detection and treatment of infected individuals is a key element of chlamydia control programs. Thus, it has been recommended that sexually active adolescents and women under 25 years of age be screened for C. trachomatis infection once per year $(6,7,26)$ or even twice per year (3-5).

The sensitivity of conventional methods for $C$. trachomatis detection based on culture or $C$. trachomatis antigen detection has been surpassed by nucleic acid amplification tests (NAATs) $(1,2,25)$. NAATs are based on the amplification of specific $C$. trachomatis target sequences by using PCR, ligase chain reaction (LCR), transcription-mediated amplification

\footnotetext{
* Corresponding author. Mailing address: Johns Hopkins University, 720 Rutland Ave., Ross 1159, Baltimore, MD 21205. Phone: (410) 614-0932. Fax: (410) 614-9775. E-mail: cgaydos@jhmi.edu.
}

(TMA), or strand displacement amplification (SDA). The amplification step allows NAATs to be significantly more sensitive than conventional methods; NAATs detect as few as one $C$. trachomatis organism per assay, whereas the limit of detection for conventional nonculture methods is 100 to 1,000 organisms $(1,25)$. NAATs are therefore considered an important advance in the field of $C$. trachomatis detection and are now in routine use for the screening and diagnosis of $C$. trachomatis.

Diagnostic assays for $C$. trachomatis may be performed with various specimens, including endocervical, vaginal, or urethral swabs and urine specimens. Compared to other collection methods, urine collection is noninvasive and is therefore much more acceptable for the individuals being tested. Urine collection is also rapid and easy and can be performed by the patients themselves, thereby avoiding the cost and time-consuming process of having a trained clinician collect each individual specimen. Thus, urine collection is regarded as a convenient method to implement in the setting of large screening campaigns for $C$. trachomatis infection.

Even though NAATs have demonstrated high sensitivities at detecting $C$. trachomatis in urine specimens $(10,11-13,15,18$, 22-24, 27), the sensitivities of these assays were often lower than with endocervical swab specimens $(10,11,21,27,29)$ and with female (versus male) urine specimens $(10,14,22,27)$. The decreased sensitivity of NAATs with urine specimens has been attributed in part to the presence of amplification inhibitors in the sample $(9,19,20)$.

The present study was carried out to compare the sensitivities of three NAATs-the Gen-Probe APTIMA Combo 2 (AC2), the Abbott LCx (based on an LCR), and BD ProbeTec 
ET (based on SDA)-for the detection of $C$. trachomatis in urine specimens from students attending school-based health clinics.

(This study was presented in part at the Infectious Disease Society of America meeting on 25 to 28 October 2001 in San Francisco, Calif.)

\section{MATERIALS AND METHODS}

Clinical sites and population. A total of 506 first-catch urine specimens were collected from asymptomatic and symptomatic males and females at various school-based health clinics as part of routine screening procedures for sexually transmitted diseases. Subject demographics and symptomatic status were not recorded for the present study. The urine samples came primarily from students in high schools and several middle schools, and the age range was 12 to 20 years. All students who present to school-based health centers for any reason are asked whether they are sexually active, and if so, they are screened, unless they refuse screening. Students seldom refuse since only a urine sample is collected and no endocervical or male urethral swabs are taken.

Specimen collection. First-catch urine specimens were self-collected. Subjects were asked not to urinate for at least $1 \mathrm{~h}$ prior to collection, and approximately $20 \mathrm{ml}$ of the initial urine stream was collected in a clean sterile container. Urine specimens were then transported and stored according to the NAAT manufacturers' specifications. Upon arrival into the laboratory, the urine samples were divided into aliquots in the transport tubes provided by each NAAT manufacturer. The identity of the sample was unlinked prior to testing by using a unique study number.

NAAT detection of $\boldsymbol{C}$. trachomatis. Specimens were tested according to the manufacturers' instructions for each assay. The AC2 assay (Gen-Probe, Inc., San Diego, Calif.) allows the detection of both C. trachomatis and Neisseria gonorrhoeae and targets $C$. trachomatis rRNA $(13,14)$. The method utilizes three steps which occur in the same tube: isolation of target sequences by using the target capture technology, amplification of isolated target sequences amplified by using TMA, and detection of the signal emitted by the hybridized probe by using a dual kinetic assay method, which allows differentiation of the signal from each pathogen.

The LCx Probe (Abbott Laboratories, Abbot Park, Ill.) is a NAAT based on LCR, which targets a sequence within the cryptic plasmid DNA of C. trachomatis. The method utilizes four steps: hybridization of probes with the target sequence, filling of gaps with the DNA polymerase and DNA ligase, amplification of the target sequence by successive reactions with the polymerase and ligase, and detection of LCR amplicons by using an immunocolorimetric bead capture system (12).

The BD ProbeTec (Becton Dickinson Diagnostic Systems, Sparks, Md.) is a NAAT based on SDA, which targets a sequence within cryptic plasmid DNA of C. trachomatis (27). The method utilizes four steps: denaturation of the target sequence, amplification of the target sequence by using the DNA polymerase and a specific restriction enzyme (SDA), and detection of the amplicons by fluorescent polarization (a fluorescent detector probe is included in the SDA reaction).

Samples which were positive only with AC2 were retested by using an alternate TMA-based NAAT, APTIMA C. trachomatis (ACT; Gen-Probe), in order to determine whether the samples were true positive or false positive. Because ACT detects a different $C$. trachomatis target sequence (located in the 16S rRNA of $C$. trachomatis), it was suitable as a confirmatory assay for AC2. In addition, for samples uniquely positive only by LCx, additional NAAT testing was performed by PCR (Roche Diagnostic Systems, Indianapolis, Ind.), since alternative target LCx-based testing was not available (28).
TABLE 1. Comparison of LCx, ProbeTec, and AC2 NAAT reactivities with 506 urine specimens

\begin{tabular}{cccc}
\hline \multirow{2}{*}{ No. of samples $^{a}$} & \multicolumn{3}{c}{ NAAT reactivity } \\
\cline { 2 - 4 } & LCx & ProbeTec & AC2 \\
\hline $69^{*}$ & + & + & + \\
0 & + & + & - \\
$3^{*}$ & + & - & + \\
$3^{*}$ & - & + & + \\
$4 \dagger$ & + & - & - \\
0 & - & + & + \\
$5 \dagger$ & - & - & - \\
$422^{\dagger}$ & - & & + \\
\hline
\end{tabular}

$a_{*}$, number of true positives (total, 75 ); $\uparrow$, number of true negatives (total, 431).

Patient infection status and data analysis. Patients were classified as infected and their specimens were considered as true positives if at least two NAATs of the original three tests were positive. The performance of each assay (including the determination of the specificity, sensitivity, positive predictive value [PPV], and negative predictive value [NPV]) was evaluated by comparing the assay's results with the infected patient status. For the sensitivity and specificity results for each assay, 95\% confidence intervals (CI) were calculated to test the significance of the estimates.

\section{RESULTS}

A total of 506 specimens from students presenting to schoolbased health clinics were collected. Although no demographic data were collected for the present study, the population was similar to that described in other studies (3-5), i.e., high-risk asymptomatic and symptomatic adolescent males and females.

Comparison of the NAAT results. Of 506 samples, 69 were positive and 422 were negative with all three assays, showing a $97 \%$ concordance between the three assays (Table 1). A total of 75 samples were positive in at least two assays (69 samples were positive in three assays, and 6 samples were positive in two assays) and were therefore considered true positives. A total of 431 samples were negative in at least two assays (422 samples were negative in three assays, and 9 samples were negative in two assays) and were therefore considered true negatives. Thus, with 75 infected individuals of 506, the calculated prevalence of $C$. trachomatis infection in this population was $14.8 \%$.

Nine samples (four in the LCx test and five in the AC2 test) were positive only in one assay out of three and were considered to be false positives. Six samples (i.e., three in ProbeTec and three in LCx) were negative in only one assay and were therefore considered to be false negatives. Thus, there were four false positives and three false negatives for $\mathrm{LCx}$, three

TABLE 2. Summary of NAAT performance with 506 urine specimens

\begin{tabular}{|c|c|c|c|c|c|c|c|c|c|c|c|}
\hline \multirow{2}{*}{ NAAT } & \multicolumn{5}{|c|}{ No. ${ }^{a}$} & \multicolumn{2}{|c|}{ Sensitivity } & \multicolumn{2}{|c|}{ Specificity } & \multirow{2}{*}{$\begin{array}{l}\text { PPV } \\
(\%)\end{array}$} & \multirow{2}{*}{$\begin{array}{c}\text { NPV } \\
(\%)\end{array}$} \\
\hline & Total & $\mathrm{TN}$ & TP & FN & $\overline{F P}$ & $\%$ & $95 \% \mathrm{CI}$ & $\%$ & $95 \% \mathrm{CI}$ & & \\
\hline $\mathrm{LCx}$ & 506 & 427 & 72 & 3 & $4 *$ & 96.0 & $88.8-99.2$ & 99.1 & $97.6-99.7$ & 94.7 & 99.3 \\
\hline ProbeTec & 506 & 431 & 72 & 3 & 0 & 96.0 & $88.8-99.2$ & 100 & $99.1-100$ & 100 & 99.3 \\
\hline $\mathrm{AC} 2$ & 506 & 426 & 75 & 0 & $5 \dagger$ & 100 & $95.2-100$ & 98.8 & $97.3-99.6$ & 93.8 & 100 \\
\hline
\end{tabular}

${ }^{a} \mathrm{TN}$, true negative; TP, true positive; FN, false negative; FP, false positive. *, two samples available for testing were both positive by PCR, which amplifies a different gene target. $\dagger$, four of these samples were confirmed as positive by AC2, a TMA-based assay, which amplifies a different gene target sequence than AC2. 
false negatives for ProbeTec, and five false positives for AC2 (Table 1 and 2).

Sensitivity, specificity, PPV, and NPV. The sensitivity and specificity for each assay were, respectively, 96.0 and $99.1 \%$ for LCx, 96.0 and $100 \%$ for ProbeTec, and 100 and $98.8 \%$ for AC2 (Table 2). Calculation of $95 \% \mathrm{CI}$ indicated that there were no statistical differences in the estimates of the sensitivity or specificity for each assay, since they all overlap (Table 2). The PPVs and NPVs were, respectively, 94.7 and $99.3 \%$ for LCx, 100 and 99.3\% for ProbeTec, and 93.8 and $100 \%$ for AC2.

Supplemental testing. Five samples were found to be positive in AC2 and negative in both LCx and ProbeTec and were therefore considered false positive in AC2 (Table 1). In order to confirm these findings, the five samples were further analyzed with an alternate NAAT, the Gen-Probe ACT, a TMAbased assay, which amplifies a different target sequence than AC2. Of the five samples retested, four were found to be positive in the alternative target NAAT, suggesting that the four samples may have been actually true positive in AC2 and false negative in the LCx and ProbeTec tests. In addition, only two of the four uniquely positive LCx samples were available for further supplemental testing by PCR. Both of these samples were positive by PCR, suggesting they may have also been true positives by LCX.

\section{DISCUSSION}

The study reported here was conducted to compare the performance of three NAATs based on different technologies (i.e., LCR, SDA, and TMA) for detecting C. trachomatis in urine specimens from high-risk adolescents. All three assays demonstrated high performance, with a sensitivity $\geq 96 \%$ and a specificity $>99 \%$. Compiled results from many studies have shown that conventional methods such as culture, enzyme immunoassay, or leukocyte esterase tests have a significantly lower sensitivity (10 to $30 \%$ less) than PCR- or LCR-based NAATs with urine samples $(1,29)$. Thus, NAATs present a great improvement in $C$. trachomatis detection with urine specimens and can be recommended for $C$. trachomatis screening programs where noninvasive and convenient self-collection is preferred. Highly sensitive assays are desired for $C$. trachomatis detection so that most infected individuals are treated in order to prevent transmission to sexual partners and to prevent serious sequelae. In the present study, the AC2 assay was slightly more sensitive than the LCR- and SDA-based assays, which each missed 3 of $75(4 \%)$ of positive samples.

This comparison study utilized the concept of a revolving "gold standard" analysis. LCx was compared against two tests as a gold standard (ProbeTec and AC2), ProbeTec was compared against LCx and AC2 as a gold standard, and AC2 was compared against LCx and ProbeTec as a gold standard. Since all tests were compared against two tests, the definition of two (or three) positive tests was used as a gold standard or "independent reference standard." The two individual tests used to define the reference standard rotated as the comparisons were performed.

Several studies have shown that the sensitivity of NAATs for C. trachomatis with urine specimens is not always optimal compared to swab samples due to the presence of amplification inhibitors or to specimen-processing procedures involving cen- trifugation which may lead to loss of sample $(9,19,20)$. In the present study and in other published studies it is probable that inhibition or sample loss may have been responsible for the false negatives observed in LCR and SDA assays. The AC2 assay yielded no false negatives in the present study, as defined by the gold standard of requiring two initially positive NAATs. The lack of inhibition in the AC2 assay has also been confirmed by others (9). The AC2 assay has been optimized to eliminate the problems related to inhibition by adding a target capture step during sample preparation to separate the target sequence from potential inhibitors (17). During the target capture process, the $C$. trachomatis-specific target sequence is hybridized with a specific capture oligomer, and the resulting complex is combined with magnetic particles. The complex comprising the target sequence, capture oligomer, and magnetic particles is subsequently separated by using a magnet and washed to remove potential amplification inhibitors. No centrifugation step is involved, which reduces the risk of losing the sample. In PCR-, SDA-, and LCR-based assays, if inhibitors are identified they can generally be inactivated by freezing, refrigerating, or diluting the sample before amplification (19, 20). However, because inhibitors are not physically removed from the sample and may resist the treatment, residual inhibition may still occur. These assays also use centrifugation steps, which may result in the loss of the urine sample pellet during aspiration, accounting for some false-negative results observed with these assays.

In addition, four of five samples, which were positive with AC2 and negative with LCx and ProbeTec, tested positive when analyzed with the ACT assay targeting a different nucleic acid sequence. This suggested that the four samples might have been true positives that were not detected by both LCx and ProbeTec. This result may have been attributed to high concentrations of inhibitors in these samples, which could not be completely inactivated during sample processing in the $\mathrm{LCx}$ and ProbeTec assays but could be completely removed by target capture in the AC2 assay. Likewise, the uniquely positive LCx samples, two of four of which were available for supplemental testing and were positive, may also be true positives that were originally missed by the ProbeTec and AC2 assays. Defining a true gold standard for positivity in the era of NAAT testing is complex and controversial, as is the need to confirm a positive NAAT test $(7,16)$.

In evaluating a new NAAT by comparison to others, there may be a tendency to underestimate specificity, since the new NAAT may be more sensitive and identify unique positives that other NAATs fail to detect (25). It is therefore crucial to further analyze unique positives with alternate methods of detection in order to determine whether they are false positives or true positives, although this approach has been controversial in the past and slightly biases the sensitivity and specificity in favor of the new test (16).

The specificity of a NAAT is critical, particularly when the test is used in a low-prevalence population. Decreased specificity in a NAAT may occur due to cross-reaction with non- $C$. trachomatis or non- $N$. gonorrhoeae organisms or to carryover contamination (7). Since false positives and decreased PPVs may be a problem in lower-prevalence populations, confirmation of positive NAAT results in clinical situations is important and is recommended now by the Centers for Disease Control 
and Prevention for lower prevalence populations (7). A confirmatory method for a NAAT should be another equally sensitive NAAT that targets a different nucleic acid sequence than the initial assay. In the present study, ACT was selected as a suitable confirmatory NAAT for AC2 because it amplifies a different target than AC2. ACT is also not likely to be affected by amplification inhibitors since, like AC2, it includes a target capture step in the sample preparation, and it did confirm four of the five unique positives detected by AC2. Similarly, since two of the four uniquely positive LCx results were confirmed by PCR, this also demonstrated the value of confirmatory testing by using a different gene target. One large concern in laboratory and public health management is the additional cost that confirmatory testing necessitates. Future studies will be required to guide public health and private practice policy in making recommendations about the need for confirmatory testing for NAAT-positive samples. Clinical judgment will also undoubtedly play a role in treatment decisions, since there is no perfect test yet. We note that, had it been possible to also test endocervical specimens in this comparison, we could have used an "infected patient standard" as the gold standard and would have probably found more positives, since tests done on endocervical swabs are usually more sensitive than those performed on urine samples. However, a limitation of this study design and a potential bias of the sensitivity estimates was that no endocervical samples were collected. It was therefore our original intent to compare the performance of the three assays with urine samples.

In summary, the LCx, ProbeTec, and AC2 assays are all highly sensitive and specific, with no differences of statistical significance in estimates of sensitivity or specificity for the noninvasive detection of $C$. trachomatis in urine specimens. Unfortunately, the LCx test is no longer commercially available, but a similar test may return in the future as a better assay. Implementation of highly sensitive NAATs for C. trachomatis detection in urine specimens represents an efficient, convenient, and cost-effective method for C. trachomatis screening in large high risk populations and should play an important role in future chlamydia control programs.

\section{ACKNOWLEDGMENTS}

We thank Florence Paillard for help in preparing the manuscript and $\mathrm{Yu}$ Hsiang Hsieh for assistance with the statistical analyses.

This study was supported in part by the kind donation of kits by Becton Dickinson and GenProbe.

\section{REFERENCES}

1. Black, C. M. 1997. Current methods of laboratory diagnosis of Chlamydia trachomatis infections. Clin. Microbiol. Rev. 10:160-184.

2. Black, C. M., J. M. Marrazzo, R. E. Johnson, E. W. Hook III, R. B. Jones, T. A. Green, J. Schachter, W. E. Stamm. G. Bolan, M. E. St. Louis, and D. H. Martin. 2002. Head-to-head multicenter comparison of DNA probe and nucleic acid amplification tests for Chlamydia trachomatis in women performed with an improved reference standard. J. Clin. Microbiol. 40:37573763 .

3. Burstein, G., C. A. Gaydos, M. Diener-West, M. R. Howell, J. Zenilman, and T. C. Quinn. 1998. Incident Chlamydia trachomatis infections among inner city adolescent females: implications for frequency of chlamydial screening. JAMA 280:521-526.

4. Burstein, G., G. Waterfield, A. Joffe, J. M. Zenilman, T. C. Quinn, and C. A. Gaydos. 1998. Screening for gonorrhea and chlamydia by DNA amplification in adolescents attending middle school health centers: opportunity for early intervention. Sex. Transm. Dis. 25:395-402.

5. Burstein, G. R., J. M. Zenilman, C. A. Gaydos, M. Diener-West, M. R. Howell, W. Brathwaite, and T. C. Quinn. 2001. Predictors of repeat Chla- mydia trachomatis infections diagnosed by DNA amplification testing among inner city females. Sex. Transmit. Infect. 77:26-32.

6. Centers for Disease Control and Prevention. 2002. Sexually transmitted diseases treatment guidelines, 2002. Morb. Mortal. Wkly. Rep. 51:1-78.

7. Centers for Disease Control and Prevention. 2002. Screening tests to detect Chlamydia trachomatis and Neisseria gonorrhoeae infections, 2002. Morb. Mortal. Wkly. Rep. 51:1-38.

8. Centers for Disease Control and Prevention. 2001. Sexually transmitted disease surveillance 2000 supplement: Chlamydia Prevalence Monitoring Project. U.S. Department of Health and Human Services, CDC, Atlanta, $\mathrm{Ga}$.

9. Chong, S., D. Jang, X. Song, J. Mahoney, A. Petrich, P. Barriga, and M. Chernesky. 2003. Specimen processing and concentration of $C$. trachomatis added can influence false-negative rates in the LCx assay but not in the APTIMA Combo 2 assay when testing for inhibitors. J. Clin. Microbiol. 41:778-782.

10. Crotchfelt, K. A., L. E. Welsh, D. DeBonville, M. Rosenstraus, and T. C. Quinn. 1997. Detection of Neisseria gonorrhoeae and Chlamydia trachomatis in genitourinary specimens from men and women by a coamplification PCR assay. J. Clin. Microbiol. 35:1536-1540.

11. Ferrero, D. V., H. N. Meyers, D. E. Schultz, and S. A. Willis. 1998. Performance of the Gen-Probe amplified Chlamydia trachomatis assay in detecting Chlamydia trachomatis in endocervical and urine specimens from women and urethral and urine specimens from men attending sexually transmitted disease and family planning clinics. J. Clin. Microbiol. 36:3230-3233.

12. Gaydos, C. A., Y. F. Ngeow, H. H. Lee, M. Canavaggio, L. E. Welsh, J. Johanson, and T. C. Quinn. 1996. Urine as a diagnostic specimen for the detection of Chlamydia trachomatis in Malaysia by ligase chain reaction. Sex. Transm. Dis. 23:402-406.

13. Gaydos, C. A., T. C. Quinn, D. Willis, A. Weissfeld, E. W. Hook, D. H. Martin, D. V. Ferrero, and J. M. Schachter. 2003. Performance of APTIMA Combo 2 assay for detection of Chlamydia trachomatis and Neisseria gonorrhoeae in female urine and endocervical swab specimens. J. Clin. Microbiol. 41:304-309.

14. Gen-Probe, Inc. 2002. Gen-Probe APTIMA Combo 2 assay for in vitro diagnostic use: user's manual. Gen-Probe, Inc., San Diego, Calif.

15. Goessens, W. H., J. W. Mouton, W. I. van der Meijden, S. Deelen, T. H. Rijsoort-Vos, N. Lemmens-den Toom, H. A. Verbrugh, and R. P. Verkooyen. 1997. Comparison of three commercially available amplification assays, AMP CT, LCx, and COBAS AMPLICOR, for detection of Chlamydia trachomatis in first-void urine. J. Clin. Microbiol. 35:2628-2633.

16. Green, T. A., C. M. Black, and R. E. Johnson. 1998. Evaluation of bias in diagnostic test sensitivity and specificity estimates computed by discrepant analysis. J. Clin. Microbiol. 36:375-381.

17. Hill, C. S. 2001. Molecular diagnostic testing for infectious diseases using TMA technology. Exp. Rev. Mol. Diagn. 1:445-455.

18. Jaschek, G., C. A. Gaydos, L. E. Welsh, and T. C. Quinn. 1993. Direct detection of Chlamydia trachomatis in urine specimens from symptomatic and asymptomatic men by using a rapid polymerase chain reaction assay. J. Clin. Microbiol. 31:1209-1212.

19. Jensen, I. P., P. Thorsen, and B. R. Moller. 1997. Sensitivity of ligase chain reaction assay of urine from pregnant women for Chlamydia trachomatis. Lancet 349:329-330.

20. Mahony, J., S. Chong, D. Jang, K. Luinstra, M. Faught, D. Dalby, J. Sellors, and M. Chernesky. 1998. Urine specimens from pregnant and nonpregnant women inhibitory to amplification of Chlamydia trachomatis nucleic acid by PCR, ligase chain reaction, and transcription-mediated amplification: identification of urinary substances associated with inhibition and removal of inhibitory activity. J. Clin. Microbiol. 36:3122-3126.

21. McCartney, R. A., J. Walker, and A. Scoular. 2001. Detection of Chlamydia trachomatis in genitourinary medicine clinic attendees: comparison of strand displacement amplification and the ligase chain reaction. Br. J. Biomed. Sci. 58:235-238.

22. Mouton, J. W., R. Verkooyen, W. I. van der Meijden, T. H. Rijsoort-Vos, W. H. Goessens, J. A. Kluytmans, S. D. Deelen, A. Luijendijk, and H. A. Verbrugh. 1997. Detection of Chlamydia trachomatis in male and female urine specimens by using the amplified Chlamydia trachomatis test. J. Clin. Microbiol. 35:1369-1372.

23. Pasternack, R., P. Vuorinen, T. Pitkajarvi, M. Koskela, and A. Miettinen. 1997. Comparison of manual Amplicor PCR, Cobas Amplicor PCR, and LCx assays for detection of Chlamydia trachomatis infection in women by using urine specimens. J. Clin. Microbiol. 35:402-405.

24. Quinn, T. C., L. Welsh, A. Lentz, K. Crotchfelt, J. Zenilman, J. Newhall, and C. Gaydos. 1996. Diagnosis by AMPLICOR PCR of Chlamydia trachomatis infection in urine samples from women and men attending sexually transmitted disease clinics. J. Clin. Microbiol. 34:1401-1406.

25. Schachter, J., and J. Moncada. 2002. Nucleic acid amplification tests to diagnose Chlamydia trachomatis genital infections: the glass is more than half full, p. 379-388. In J. Schachter, G. Chirtiansen, I. N. Clarke, M. R. Hammerschlag, B. Kaltenboeck, C. C. Kuo, R. G. Rank, G. L. Ridgway, P. 
Saikku, W. E. Stamm, R. S. Stephens, J. T. Summersgill, P. Timms, and P. B Wyrick (ed.), Chlamydial infections. Proceedings of the 10th International Symposium on Human Chlamydial Infections. GRAFMAT Basim ve Reklam Sanayi Tic. Ltd. Sti., Antalya, Turkey.

26. U.S. Preventive Services Task Force. 2001. Screening for chlamydial infection, recommendations and rationale. Am. J. Prev. Med. 20:90-94.

27. Van Der Pol, B., D. V. Ferrero, L. Buck-Barrington, E. Hook III, C. Lenderman, T. Quinn, C. A. Gaydos, J. Lovchik, J. Schachter, J. Moncada, G. Hall, M. J. Tuohy, and R. B. Jones. 2001. Multicenter evaluation of the BDProbeTec ET System for detection of Chlamydia trachomatis and Neis- seria gonorrhoeae in urine specimens, female endocervical swabs, and male urethral swabs. J. Clin. Microbiol. 39:1008-1016.

28. Van Der Pol, B., T. C. Quinn, C. A. Gaydos, K. Crotchfelt, J. Schachter, J. Moncada, D. Jungkind, D. H. Martin, B. Turner, C. Peyton, and Robert B. Jones. 2000. Multi-center evaluation of the AMPLICOR and automated COBAS AMPLICOR CT/NG tests for the detection of Chlamydia trachomatis. J. Clin. Microbiol. 38:1105-1112.

29. Watson, E. J., A. Templeton, I. Russell, J. Paavonen, P.-A. Mardh, A. Stary, and B. S. Pederson. 2002. The accuracy and efficacy of screening tests for Chlamydia trachomatis: a systematic review. J. Med. Microbiol. 51:1021-1031. 